\title{
Persistent Left Superior Vena Cava: A Finding After Central Venous Catheterization
}

\author{
Francisco Lopes Morgado, Bárbara Saraiva, Celestina Blanco Torres, João Correia \\ Internal Medicine Department, Unidade Local de Saúde da Guarda, Guarda, Portugal
}

Received: $13 / 12 / 2020$

Accepted: 23/12/2020

Published: $21 / 01 / 2021$

\begin{abstract}
How to cite this article: Lopes Morgado F, Saraiva B, Blanco Torres C, Correia J. Persistent left superior vena cava: a finding after central venous
\end{abstract} catheterization. EJCRIM 2021;8: doi:10.12890/2020_002161.

Conflicts of Interests: The Authors declare that there are no competing interests.

Acknowledgements: We gratefully acknowledge our patient and everyone involved in her treatment.

This article is licensed under a Commons Attribution Non-Commercial 4.0 License

\section{ABSTRACT}

Introduction: Persistence of the left superior vena cava (LSVC) is a rare anatomical variant in the general population, with an estimated incidence of $0.3-0.5 \%$ in healthy individuals. It may be diagnosed incidentally after imaging control of the placement of a central venous catheter (CVC) or other device.

Patient and Methods: We present the case of a patient with acute disease who required central venous catheterization for the administration of intravenous chemotherapy.

Results: Central venous catheterization proved difficult and imaging control revealed the catheter tip in an unusual position. Computed tomography to verify the catheter tip position revealed the presence of a persistent left superior vena cava. The patient then underwent the planned treatment with no complications associated with the CVC.

Conclusion: Although uncommon, persistence of the LSVC can have a significant impact in clinical practice, particularly when invasive procedures are required. Its recognition is important in order to minimize the potential complications inherent to such procedures.

\section{LEARNING POINTS}

- Persistence of the left superior vena cava is an uncommon anatomical variant of the central venous vascular anatomy.

- It is generally asymptomatic, and its diagnosis is often incidentally made after certain procedures (central venous catheterization).

- Diagnosis through chest $\mathrm{x}$-ray alone is difficult and should be complemented with transthoracic echocardiography and computed tomography in order to minimize the complications inherent to central venous catheterization.

\section{KEYWORDS}

Superior vena cava, central venous catheterization

\section{INTRODUCTION}

Persistence of the left superior vena cava (LSVC) is a relatively rare anatomical variant in the general population, with an estimated incidence of $0.3-0.5 \%$ in healthy individuals ${ }^{[1,2]}$. As it is normally asymptomatic, diagnosis may be incidental after imaging control of the placement of a central venous catheter (CVC) or other device ${ }^{[1,2]}$. Its documentation is important since it may have clinical implications in specific cases, such as continuous haemodynamic monitoring or the correct positioning of a pacemaker. We present the case of a patient with persistent LSVC diagnosed after CVC placement during acute pathology. 


\section{CASE DESCRIPTION}

A 70-year-old woman, diagnosed with acute myeloid leukaemia, was urgently admitted to begin intravenous induction chemotherapy. The first attempt to place a CVC was made in the right subclavian vein, with difficulty in the progression of the guidewire. After successful placement, a control chest x-ray was performed, and the tip of the catheter was seen in the homolateral jugular vein.

Given the need to start cytotoxic therapy, a new attempt was made to place a CVC, this time in the left subclavian vein. The procedure was carried out without complications; however, radiology showed the catheter was positioned to the left of the cardiac shadow, overlapping the image of the aortic artery (Fig. 1). Blood was collected from the CVC for gas analysis and was compatible with venous blood. Because of doubt regarding the position of the catheter, thoracic computed tomography was performed, which confirmed catheterization of an LSVC (Figs. 2 and 3).
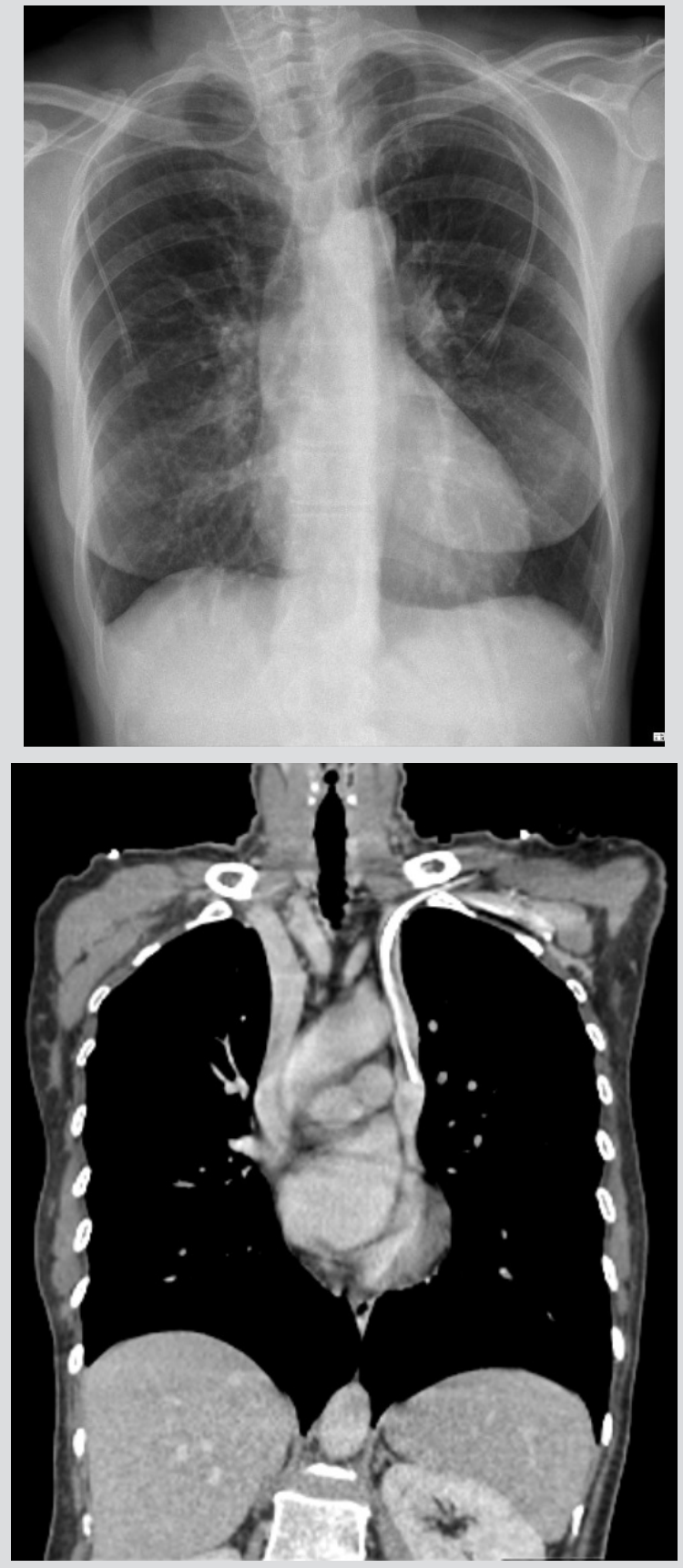

Figure 2. CT scan (coronal view) showing catheterization of the left superior vena cava. The regular superior vena cava can also be seen on the right
Figure 1. Chest radiogram showing the presence of both central venous catheters (CVCS) described in this case. Migration of the right subclavian CVC to the jugular vein can be seen. The left subclavian CVC has an unusual trajectory, with the tip positioned to the left side of the heart

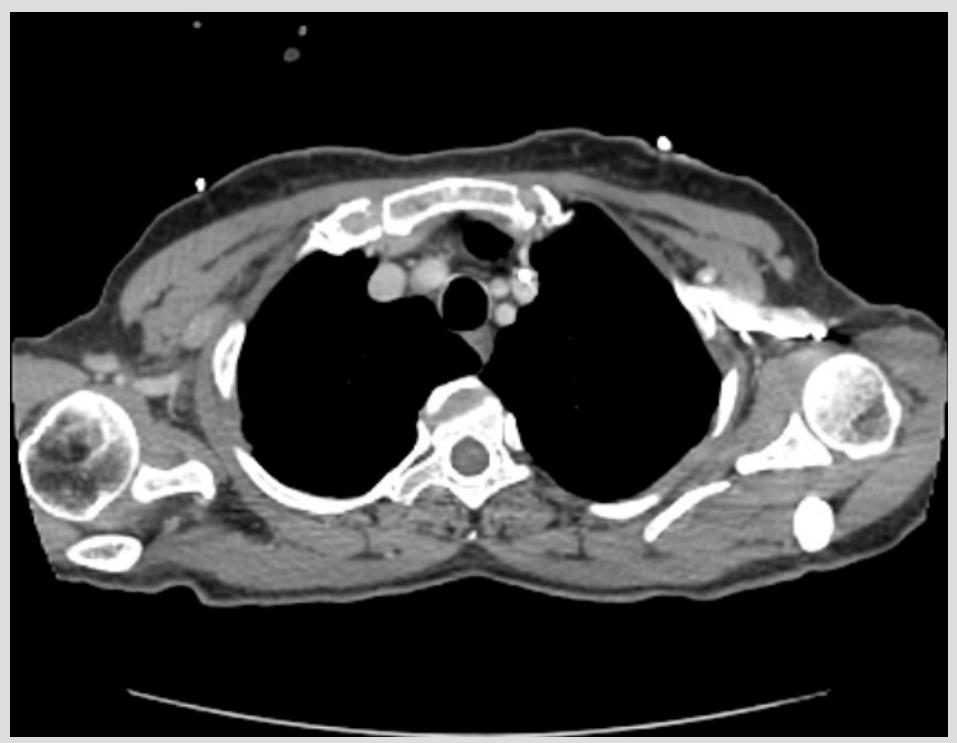

Figure 3. CT scan (axial view) showing the catheterized persistent left superior vena cava 
The patient was then submitted to the planned treatment and discharged on the 35th day of hospitalization without complications associated with the CVCs.

\section{DISCUSSION}

All professionals who perform invasive procedures should be familiar with central venous vascular anatomy. Persistent LSVC, despite its rarity, is the most frequent anatomical variant of the thoracic venous system ${ }^{[1,2]}$ and is caused by persistence of the left common cardinal vein $^{[2]}$. In most cases, the LSVC drains into the coronary sinus of the right atrium with a concomitant normal right superior vena cava, which can be connected by a smaller calibre accessory vein ${ }^{[2]}$. Despite being generally asymptomatic, patients are more susceptible to cardiac arrhythmias due to anomalies in the atrioventricular node and the bundle of His. In the rare cases (<8\%) where venous drainage is into the left heart, patients may show symptomatic right-to-left shunt ${ }^{[1,2]}$.

The diagnosis of LSVC persistence with chest radiography alone is difficult, however, there may be some suggestive signs, such as an abnormal outline of the upper mediastinum caused by the widening of the aortic shadow ${ }^{[2]}$. Transthoracic echocardiography can also be a useful tool, sometimes identifying an enlarged coronary sinus ${ }^{[3]}$. Computed tomography, especially with intravenous contrast, is the best method for visualization, allowing a detailed assessment of the thoracic vasculature and confirmation ${ }^{[4]}$ of the exact location of the CVC tip as shown in this case (Fig. 2).

\section{CONCLUSION}

Although in most cases persistent LSVC has no clinical impact, the internist must be alert to its existence, particularly when subclavian or jugular central venous catheterization is difficult. The degree of suspicion must be high in order to minimize the serious potential complications inherent to this type of procedure.

\section{REFERENCES}

1. Gibson F, Bodenham A. Misplaced central venous catheters: applied anatomy and practical management. Br J Anaesth 2013;110(3):333-346.

2. Schummer W, Schummer C, Fröber R. Persistent left superior vena cava and central venous catheter position: clinical impact illustrated by four cases. Surg Radiol Anat 2003;25:315-321.

3. Goyal SK, Punnam SR, Verma G, Ruberg FL. Persistent left superior vena cava: a case report and review of literature. Cardiovasc Ultrasound 2008;6:50.

4. Mneimneh S, Shatila A, Shehab G, Rajab M. Persistent left superior vena cava: a case report and review of literature. Int J Clin Trials 2014;1(3):114-116. 